\title{
Interventions en supervision directe pour développer la compétence du résident : une recherche qualitative
}

\author{
Interventions in direct supervision for developping \\ resident competence: a qualitative study
}

Bernard MARTINEAU', Gilles GIRARD' , Richard BOULE'

\begin{abstract}
Résumé Contexte : La littérature médicale sur la supervision directe a davantage exploré les contenus abordés en supervision que les stratégies utilisées par les superviseurs pour atteindre les objectifs pédagogiques. St-Arnand a proposé un modèle intégré de stratégies pour développer la coopération en relation d'aide. Objectif: Cet article vise à tester le modèle de St-Arnaud en formation médicale et à identifier les stratégies de superviseurs experts en supervision directe pour maintenir une relation de coopération et faciliter les apprentissages professionnels de résidents en médecine de famille. Méthodes: Ces stratégies ont été identifiées à l'aide d'une recherche exploratoire qualitative basée sur une série d'études de cas. Trois superviseurs experts, reconnus par leurs pairs, ont été observés durant trois demi-journées de supervision avec trois résidents chacun. Le verbatim des entretiens a été codé et analysé par deux chercheurs selon le schéma d'analyse de St-Arnaud. Après chaque session, le superviseur et le résident ont été interviewés individuellement sur les stratégies du superviseur. Résultats : Quatre niveaux complémentaires d'intervention sont mis en évidence durant la supervision: 1. gestion du processus de supervision; 2. résolution du problème à travers la compétence du résident; 3. résolution du problème selon l'expertise du superviseur; 4. démarche réflexive du résident. Diverses stratégies et procédés sont utilisés à chaque niveau d'intervention et aux différentes étapes de la supervision. Conclusion: Les niveaux d'intervention et les stratégies aident les superviseurs à favoriser la collaboration et les apprentissages du supervisé. Lidentification de ces stratégies facilitera la formation des superviseurs.
\end{abstract}

Mots clés Education médicale ; stratégies d'interventions; supervision directe.

Abstract Context: The literature based on direct supervision explore more the supervision content per se than strategies used by supervisors to reach educational objectives. St-Arnaud has proposed a model, which integrates strategies to develop cooperation in the context of assistance. Objective: In this study, our goals are to test St-Arnand's model in the course of medical formation of first year residents in family medicine and to identify strategies used by expert supervisors during direct supervision in order to maintain a cooperative relationship with residents and to facilitate their learning. Methods: These strategies have been identified by the means of an exploratory qualitative research based on several case studies. Three expert supervisors, which were acknowledged by their pairs, have been observed during three half days of supervision with three residents each. Sessions' verbatim was coded and analyzed according to the St-Arnaud analysis scheme by two researchers. After each session, the supervisor and the resident were individually interviewed to discuss the supervisor' strategies. Results: Four complementary levels of intervention are identified during supervision sessions; 1) management of the supervision process; 2) problem solving according to the resident' competency; 3) problem solving according to the supervisor' expertise and 4) resident' reflective practice. Diverse strategies and processes were used at each level of the intervention and at different steps of the supervision. Conclusion: Intervention levels and strategies help supervisors to favour collaboration with residents and to facilitate their learning. The identification of these strategies will facilitate supervisors' formation.

Key words Medical education; strategies of interventions; direct supervision.

Pédagogie Médicale 2008;9:19-31 


\section{Recherche et Perspectives}

\section{Introduction}

Kilminster et ses collaborateurs ${ }^{1,2}$ définissent la supervision clinique comme étant la fourniture d'une guidance à l'égard d'un étudiant en formation et d'une rétroaction (feedback) à l'égard de son développement personnel, professionnel et éducationnel, dans le contexte d'une expérience de soins dispensés à un patient avec sécurité et de manière appropriée. La supervision clinique est reconnue dans la littérature ${ }^{1,2}$ comme étant à la fois essentielle et efficace pour la formation du médecin. Toutefois, une grande disparité dans la qualité et la fréquence des supervisions est rapportée selon les milieux de formation et les spécialités concernées ${ }^{1,2}$. On distingue différentes modalités de supervision : a) la "supervision strictement clinique " qui cible l'efficacité des pratiques médicales et la sécurité du patient et b) la "supervision pédagogique en contexte clinique " qui, outre l'objectif précédent, vise explicitement à faciliter, à contextualiser et à orienter les apprentissages à effectuer par l'étudiant supervisé. Ces deux modalités sont souvent implicitement liées, mais elles ont avantage à être explicitement articulées, car elles requièrent l'une et l'autre des compétences spécifiques. A un autre niveau, les modalités de supervision adoptées permettent de distinguer : a) la "supervision avec observation directe et feedback immédiat ou à court terme de la part du superviseur »-que nous désignerons communément par le terme de "supervision directe " dans la suite du texte et b) la «supervision sans observation directe et avec feedback différé " lorsque le superviseur, absent au moment de la pratique clinique, donne son feedback à partir de la présentation du cas par l'apprenant.

Des données probantes établies à partir de recherches empiriques $^{1,2}$ démontrent que la supervision pédagogique en contexte clinique développée à partir de l'observation directe de l'étudiant en situation professionnelle influence positivement la qualité des soins donnés au patient et le développement de l'apprenant, spécialement lorsqu'elle comporte un feedback ciblé. La littérature met en évidence que l'efficacité de la supervision dépend principalement de la qualité de la relation entre le superviseur et le résident ${ }^{2-4}$. Dans la littérature médicale sur la supervision directe, les contenus abordés ${ }^{5,6}$ ont été davantage explorés que les stratégies utilisées par les superviseurs pour atteindre leurs objectifs éducatifs. Toutefois, Westberg et Jason ${ }^{7}$ ont souligné les avantages qu'il y avait à promouvoir l'autoréflexion de l'apprenant et à donner un feedback efficace en éducation médicale. Plusieurs auteurs ${ }^{8,9}$ ont précisé les conditions d'un feedback efficace. Par ailleurs, Irby ${ }^{10}$ insiste sur l'importance de la collaboration superviseur-supervisé dans le cadre de l'activité de supervision, démontrant son impact direct sur la capacité de résolution de problème de l'apprenant et la qualité des soins au malade. De plus, Huba and Freed ${ }^{11}$ décrivent diverses techniques de questionnement, dont celles formalisées par Christensen ${ }^{12}$, pour favoriser l'apprentissage en supervision. Un modèle intégré des stratégies efficaces d'intervention du superviseur en formation médicale reste toutefois à préciser.

St-Arnaud ${ }^{11-13}$ a développé et validé un modèle intégré de stratégies d'intervention en supervision de la relation d'aide. Il a mis en évidence que l'efficacité de l'interaction professionnelle augmente dans la mesure où le professionnel réussit à établir et à maintenir une relation de coopération avec la personne qui le consulte. Pour St-Arnaud, la coopération n'est jamais acquise au point de départ d'une relation et relève, dans une relation professionnelle, de la compétence de l'intervenant. Dans le but d'être en mesure d'apporter un soutien à une telle relation, il a formulé et illustré cinq règles pratiques qui permettent de constater l'efficacité de la coopération dans la relation superviseur-supervisé : le partenariat, la concertation, l'alternance, la non-ingérence et la responsabilisation. En conséquence, la présente étude vise à tester et à ajuster, s'il y a lieu, le modèle de St-Arnaud dans le contexte de la supervision en formation médicale et, plus particulièrement, à identifier les stratégies d'interventions utilisées en supervision directe de résidents en médecine de famille par des superviseurs experts en vue de créer et maintenir une relation de coopération avec le résident et de faciliter ses apprentissages professionnels.

\section{Méthodes}

Comme le domaine de l'interaction superviseur-supervisé a été peu exploré en médecine, nous avons opté pour une recherche qualitative exploratoire sur la base d'une série d'études de cas ${ }^{16}$. Avant d'exposer la méthode mise en œuvre pour notre travail, il est impor-

1- Département de médecine de famille, Faculté de médecine, Université de Sherbrooke.

Correspondance : Bernard Martineau. Département de médecine de famille, Faculté de médecine, Université de Sherbrooke, 3001-12e Avenue Nord, Sherbrooke, Québec, Canada J1H 5N4. Téléphone : (819) 346-1110, extension 14273 - Télécopieur : + 1 (819) 564-5386. Mailto:Bernard.Martineau@USherbrooke.ca 
tant de préciser le contexte de l'étude. Au Canada, la formation post-graduée en médecine familiale dure deux ans. La supervision directe est une méthode d'enseignement utilisée de manière systématique dans les milieux de formation en médecine familiale. Durant leurs consultations avec les patients, les étudiants sont souvent observés, derrière un miroir sans tain, par un médecin seul ou accompagné d'un intervenant psychosocial. A la fin de la période de consultation ou après l'entrevue avec chaque patient, les observateurs donnent une rétroaction à l'étudiant pour améliorer sa compétence professionnelle. La session de supervision comporte la préparation, l'observation directe et l'entretien de feedback.

Afin d'identifier un éventail de stratégies d'interventions efficaces, les deux premiers chercheurs (BM, GG) ont observé trois superviseurs experts, reconnus par leurs pairs, durant une demi-journée de supervision directe avec au moins trois résidents différents chacun ou jusqu'à saturation des données. Ces trois médecins de famille sont des enseignants cliniciens experts, tels que définis par Chamberland et Hivon ${ }^{17}$, qui enseignent depuis plus de quinze ans. Ils ont aussi été sélectionnés pour leur capacité réflexive et leur habilité à verbaliser leur démarche. Pour des raisons pratiques, les deux chercheurs principaux (BM, GG) ont choisi les superviseurs experts dans le milieu même où ils travaillent et qui comprend une vingtaine de superviseurs. À la fin de la demi-journée, des entrevues semi-structurées de 1520 minutes ont été effectuées individuellement avec le superviseur et le résident, pour recueillir leurs commentaires libres sur la supervision, puis leur permettre d'en préciser les moments clés, de même que leurs objectifs en cours de session, les stratégies utilisées pour y répondre et les messages retenus de la supervision. Les étu- diants étaient volontaires pour participer à cette recherche. Les patients étaient informés de la présence et du rôle du superviseur et des deux chercheurs derrière le miroir. Ils ont donné leur accord mais n'étaient pas concernés par l'objet de la recherche qui est l'étude de la relation superviseur-supervisé. Le comité d'éthique de notre faculté de médecine a entériné notre projet. L'enregistrement audio de la supervision et des entrevues semi-structurées a été transcrit de manière intégrale. Cette transcription a servi pour l'analyse des données. La collecte des données des neuf supervisions étudiées a été effectuée sur deux ans entre 2003 et 2005. Les neuf résidents étaient en première année de formation durant leur semestre de médecine familiale. Durant les demi-journées étudiées, les étudiants voyaient en général trois patients.

De même que le cadre conceptuel, le cadre d'analyse choisi s'inscrit dans une démarche praxéologique ( $c f$. ci-dessous) inspirée d'Argyris, Schön et St-Arnaud ${ }^{13-15,18,19}$. C'est une démarche de recherche-action où les interventions des superviseurs experts, de même que leurs réflexions sur leurs actions pédagogiques, sont confrontées aux observations des chercheurs. La grille d'interaction professionnelle de St-Arnaud, comprenant 12 stratégies et 32 procédés, a servi de base à l'analyse pour rechercher les stratégies spécifiques utilisées par les superviseurs experts durant la supervision directe. Chaque intervention des superviseurs était cotée et catégorisée indépendamment par deux chercheurs qui discutaient au besoin leurs cotations et s'entendaient sur un consensus. Ils ont donc effectué une approche déductive à partir du système de cotation développé par St-Arnaud qui a agi comme expert externe pour valider les cotations et catégorisations des chercheurs. Les entrevues semi-structurées de même que les notes du superviseur ont été classifiées de la même manière. À l'aide du test personnel

La praxéologie est une science de l'action humaine qui vise à comprendre l'agir professionnel dans ses pratiques quotidiennes, dans ses processus de changement et dans la mesure de ses conséquences. Dès le moment où l'on essaie de résoudre et comprendre une situation problématique en s'efforçant d'observer l'effet de ses propres actions pour être en mesure de les modifier au moment de faire de nouvelles actions, on devient un praticien réflexif. Le terme réflexion dans l'action est utilisé pour décrire le processus mental qui permet à un professionnel de s'adapter à chaque situation où il exerce sa profession. La réflexion dans l'action est une sorte de dialogue continuel entre le praticien et les événements de sa pratique professionnelle. En apprenant à utiliser systématiquement la réflexion dans l'action, le praticien peut augmenter de façon significative l'efficacité de ses interventions. Il peut aussi développer progressivement une sorte de modèle d'intervention sur mesure $^{13}$. 


\section{Recherche et Perspectives}

d'efficacité de St-Arnaud ${ }^{14}$, qui consiste à évaluer si le superviseur adapte ses stratégies en fonction de l'effet produit chez le supervisé, les réponses des résidents supervisés ont été considérées pour déterminer leur niveau d'accord avec les interventions du superviseur. L'analyse des données était d'abord verticale, visant à explorer à fond chaque entretien, puis horizontale pour rechercher une cohérence entre les entretiens. Les chercheurs ont pu ainsi déterminer l'efficacité des stratégies utilisées par les superviseurs et le niveau de collaboration atteint dans la supervision. Pour s'assurer que l'analyse représentait bien le vécu des superviseurs experts et dans un objectif de validation par triangulation des données, les résultats ont été présentés aux superviseurs experts, une fois l'analyse terminée, pour savoir s'ils se reconnaissaient dans la description de leurs stratégies formulée par les chercheurs.

Les deux chercheurs principaux (un médecin de famille détenteur d'une maîtrise en pédagogie et un psychologue) sont des formateurs impliqués en médecine de famille depuis plus de vingt ans. Le troisième chercheur (RB) est l'un de nos trois superviseurs experts qui s'est joint aux deux premiers auteurs une fois que la cotation et l'analyse de ses propres supervisions furent effectuées par les deux autres chercheurs. De plus, il n'a pas participé au recueil des données des autres superviseurs. Il a contribué à la validation de l'analyse des données et à la rédaction du présent article. Cette forme de validation par triangulation (deuxième regard par les personnes impliquées dans l'action) est encouragée en praxéologie.

\section{Résultats}

Trois axes ressortent de l'analyse : les niveaux d'intervention choisis par le superviseur, les champs d'intervention fortement imprégnés de la démarche médicale et les stratégies et procédés utilisés pour mettre en œuvre les objectifs des superviseurs. Notre échantillon d'interactions superviseur-supervisé nous a permis d'atteindre rapidement une saturation des données. Les mêmes niveaux et champs d'intervention de même que les mêmes stratégies et procédés se retrouvent dans les interventions des trois superviseurs, bien que le profil individuel de leurs interventions varie selon le style de chacun, les besoins du résident et la réalité clinique du patient. De plus, après l'analyse des interactions entre les superviseurs et deux résidents, toutes les stratégies et les procédés décrits étaient répertoriés. L'analyse des interactions de chacun des superviseurs avec un troisième résident n’a permis que de confirmer ce qui était déjà identifié. De courts segments d'interventions du superviseur illustreront les différents concepts. Enfin, les constats sur les niveaux de collaboration seront détaillés et le déroulement d'une demi-journée de supervision directe évoqué.

\section{Niveaux d'intervention (tableau 1)}

Quatre niveaux complémentaires d'intervention sont identifiés durant la supervision directe. Le plus souvent, le superviseur expert les utilise de façon itérative dans la séquence décrite. Il débute la supervision par des interventions au niveau de la gestion du processus (niveau 1), qu'il réitère chaque fois qu'il réoriente le dialogue par une signalisation du déroulement de la supervision. Avant les rencontres avec les patients, il précise avec le résident supervisé des objectifs d'apprentissage et propose un déroulement pour optimiser la supervision. D'emblée, après chaque entrevue, il questionne d'abord le résident sur son point de vue et son fonctionnement (niveau 2) en portant attention à la cohérence de son raisonnement. Il favorise une position de facilitation dans le développement de la compétence des résidents avant de suppléer ses déficiences. Toutefois, au besoin, le superviseur complète les éléments manquants (niveau 3) grâce à son expertise à la fois clinique et pédagogique. Il incite alors le supervisé à se les approprier. Finalement, selon les difficultés rencontrées et le temps disponible, il offre au résident d'amorcer une démarche réflexive (niveau 4), qui va au-delà du contexte de la consultation, pour que ce dernier puisse évoluer dans sa résolution des problèmes au prix d'une remise en question de ses façons de voir.

\section{Champs d'intervention (tableau 1)}

Par champs d'intervention, on réfere aux contenus des interventions du superviseur pour favoriser le développement de la compétence du supervisé, indépendamment du niveau choisi. Trois types de contenus sont ciblés, tant au plan clinique que pédagogique : A) la formulation du problème présenté par le patient (plan clinique) ou le supervisé (plan pédagogique) ; B) le choix des objectifs d'intervention par rapport aux problèmes soulevés et $\mathrm{C}$ ) les moyens d'investigation ou d'intervention qui seront privilégiés pour le patient ou la formation du supervisé. Ces champs d'interventions s'apparentent aux étapes de la démarche clinique qui sont de poser un diagnostic, d'opter pour des objectifs d'intervention et finalement de choisir et de mettre en œuvre des moyens d'investigation et de traitement. On constate que le champ du choix des objectifs est moins exploité par nos superviseurs aux niveaux " résolution 


\begin{tabular}{|c|c|c|c|c|c|}
\hline \multicolumn{6}{|c|}{$\begin{array}{l}\text { Tableau } 1 \text { : } \\
\text { Illustration des niveaux et des champs d'intervention }\end{array}$} \\
\hline & & & \multicolumn{3}{|c|}{ Champs d'intervention } \\
\hline & & & $\begin{array}{l}\text { A. Formulation } \\
\text { du problème }\end{array}$ & B. Choix d'objectifs & $\begin{array}{c}\text { C. Moyens } \\
\text { d'investigation ou } \\
\text { d'intervention }\end{array}$ \\
\hline \multirow{4}{*}{ 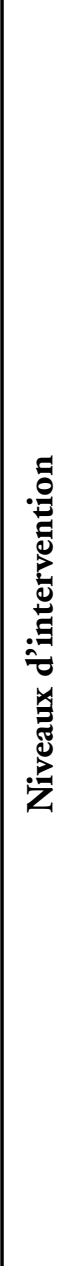 } & 1 & $\begin{array}{l}\text { Gestion du processus } \\
\text { de supervision }\end{array}$ & $\begin{array}{l}\text { "Je te propose de régler } \\
\text { le cas clinique. Quand } \\
\text { tu auras libéré ton } \\
\text { patient, nous discute- } \\
\text { rons de l'entrevue." }\end{array}$ & $\begin{array}{l}\text { "Je te propose de } \\
\text { modifier notre cible } \\
\text { initiale..." }\end{array}$ & $\begin{array}{l}\text { "Je te propose un mini } \\
\text { jeu de rôle où je jouerai } \\
\text { le rôle de ton patient." }\end{array}$ \\
\hline & 2 & $\begin{array}{l}\text { Résolution du } \\
\text { problème à travers } \\
\text { la compétence du } \\
\text { résident }\end{array}$ & $\begin{array}{l}\text { "Selon toi, quels sont } \\
\text { les problèmes du } \\
\text { patient? " Quelles sont } \\
\text { tes hypothèses diagnos- } \\
\text { tiques?" }\end{array}$ & $\begin{array}{l}\text { "Quel est ton objectif } \\
\text { avec ce patient?" }\end{array}$ & $\begin{array}{l}\text { "Qu'est-ce que tu } \\
\text { comptes faire avec ce } \\
\text { patient?" }\end{array}$ \\
\hline & 3 & $\begin{array}{l}\text { Résolution du } \\
\text { problème selon } \\
\text { l'expertise du } \\
\text { superviseur }\end{array}$ & $\begin{array}{l}\text { «Selon moi, le patient } \\
\text { présente..." }\end{array}$ & $\begin{array}{l}\text { "Selon moi, le pro- } \\
\text { chain pas avec ce } \\
\text { patient est..." }\end{array}$ & $\begin{array}{l}\text { "Selon moi, tu devrais } \\
\text { faire tel examen ou tel } \\
\text { test... et envisager tel } \\
\text { traitement." }\end{array}$ \\
\hline & 4 & $\begin{array}{l}\text { Démarche réflexive } \\
\text { du résident }\end{array}$ & $\begin{array}{l}\text { "Qu'est-ce qui motive } \\
\text { tes hypothèses diagnos- } \\
\text { tiques?" "Qu'est-ce qui } \\
\text { t'empêche d'envisager } \\
\text { telle hypothèse?" }\end{array}$ & $\begin{array}{l}\text { "Qu'est-ce qui fait que } \\
\text { tu n'as pas parlé d'ob- } \\
\text { jectif avec ce patient?" }\end{array}$ & $\begin{array}{l}\text { "Comment expliques- } \\
\text { tu que tu hésites à } \\
\text { prescrire tel traitement } \\
\text { pour ce patient?" }\end{array}$ \\
\hline
\end{tabular}

de problème à travers la compétence du résident " et "démarche réflexive " (tableau 2). A ces niveaux, les superviseurs passent directement du problème aux solutions. Par ailleurs, c'est lorsqu'ils utilisent leur expertise pour résoudre les problèmes que les superviseurs investissent le plus au niveau des objectifs en transmettant de nombreuses connaissances pour orienter les résidents. Ce sont en général les interventions les plus longues.

\section{Stratégies utilisées (tableau 2)}

Les stratégies et les procédés utilisés par les superviseurs pour potentialiser la supervision tiennent compte à la fois du niveau et du champ d'intervention choisis. Les 12 grandes stratégies décrites par St-Arnaud ${ }^{15}$ dans le cadre de la relation d'aide (fond gris dans le tableau), trois par niveau d'intervention, se retrouvent dans les interventions des superviseurs experts. C'est au niveau 


\section{Recherche et Perspectives}

des 22 procédés ou modalités d'application de ces stratégies (fond blanc) que l'on note des particularités en supervision médicale. Les procédés suivants sont nouveaux par rapport au modèle initial de St-Arnaud : tester les connaissances et vérifier la compréhension du problème du patient (niveau 2), aider au diagnostic différentiel et donner des exemples pour illustrer une résolution de problème (niveau 3 ) et explorer la démarche clinique (niveau 4).

Le procédé le plus fréquemment utilisé par nos superviseurs experts est la " réception manifeste " ou l'accueil de ce que dit le supervisé, soit aux niveaux 2 et 4 , lorsque le superviseur cherche à développer la compétence du supervisé, en l'interrogeant sur les faits, son raisonnement et son expérience. La réception manifeste se traduit par un silence attentif du superviseur au moment où le résident fait une pause et par des facilitateurs du type "hum-hum, ok, oui, jécoute " qui invitent le résident à poursuivre son élaboration. Par ailleurs, au niveau de la résolution du problème selon l'expertise du superviseur (niveau 3), « renseigner » ou transmettre des informations médicales manquantes pour résoudre le problème du patient, " donner un feedback spécifique " au supervisé et " émettre une opinion d'expert, suggérer des diagnostics différentiels et approuver ou donner son accord " sont également parmi les procédés les plus utilisés en lien avec les stratégies consistant à évaluer, orienter et habiliter. Bien que moins fréquentes, d'autres stratégies telles que la "structuration de la démarche, l'encadrement du dialogue (niveau 1) et l'invitation à l'auto-critique " (niveau 4) sont présentes à chaque supervision. Enfin, d'autres stratégies telles que « l'instrumentation dans la relation à l'aide d'exercices, l'anticipation et la planification des interventions et la stimulation de la créativité à travers l'exploration des impacts de divers choix thérapeutiques " sont utilisées occasionnellement selon la nécessité de résoudre le problème du patient, les besoins manifestés par le résident, le temps disponible et le style du superviseur.

\section{Relation de collaboration}

Les neuf supervisions ont été analysées à la lumière des cinq règles ou repères pratiques de coopération de St-Arnaud énoncés dans l'introduction. Voici les principaux constats.

Pour ce qui est du partenariat qui consiste en la poursuite d'un but commun, on constate que les trois superviseurs prennent un temps en début de demi-journée pour discuter de la cible visée en supervision et de la manière de fonctionner pour l'atteindre. Ils emploient souvent les mots "nous " et " ensemble " dans leurs échanges avec les supervisés. En lien avec le partenariat, la concertation consiste à vérifier qu'on poursuit une même cible à chaque moment du dialogue. Durant les échanges, le superviseur annonce régulièrement ses intentions et obtient l'accord du supervisé sur le processus, particulièrement lorsqu'il l'invite à s'auto-évaluer (niveau 4). Exemple: En présence d'un résident qui éprouve des difficultés à porter attention aux préoccupations de ses patients et à les faire élaborer sur ce qui les préoccupe, le superviseur lui propose: "Si tu es d'accord, discutons brièvement de ce qui t'empêche de faire travailler tes patients davantage en entrevue?" Donner ainsi au résident le choix de s'engager ou non dans ce qui lui est proposé, augmente son sentiment de liberté et le responsabilise par rapport à son cheminement. Un des superviseurs experts ne sollicitait pas d'emblée la permission d'ouvrir sur des sujets plus personnels concernant le supervisé ; il l'a fait par la suite en constatant les difficultés soulevées chez le supervisé.

L'alternance est une intervention à deux composantes pour faciliter la coopération : d'une part, la réception du message du supervisé par une reformulation, un reflet ou un résumé et, d'autre part, une direction ou une consigne indiquée au supervisé par rapport à ce qu'on attend de lui à ce moment de l'entretien (demande d'élaboration ou de précision, explication, signalisation sur le processus). Exemple: "J'entends que tu as de la difficulté à discuter de la perspective du patient. Je te propose de commencer par rechercher les impacts du problème présenté par le patient». Les superviseurs experts utilisent l'alternance dans plus de $40 \%$ de leurs interventions : ce taux est reconnu comme facilitant la coopération selon les standards praxéologiques de St-Arnaud ${ }^{14}$.

La non-ingérence se définit comme la reconnaissance par le superviseur des limites de son pouvoir lorsqu'il veut amener un changement chez le supervisé. On constate l'absence de jugements de valeur dans les interventions de nos superviseurs. Ils privilégient les choix personnels du supervisé dans la mesure où ils n'interferent pas avec le bien-être du patient. Par ailleurs, la responsabilisation suppose l'invitation explicite du superviseur au supervisé à faire des choix personnels, à s'impliquer dans le processus de soins. Les trois superviseurs invitent régulièrement les supervisés à mettre à profit leurs capacités et recherchent d'abord leur avis au lieu de leur prescrire d'emblée une conduite dont ils 
seraient les simples exécutants. Exemple: Une résidente termine une entrevue avec une patiente de 27 ans chez laquelle elle a évalué un début de première grossesse normale, mais a négligé ses inquiétudes par rapport au fait qu'elle a souffert d'une dépression il y a deux ans. Le superviseur valide d'abord ce qu'elle a réussi à faire avec cette patiente et l'invite à prévoir comment elle discutera avec elle, lors d'une prochaine rencontre, de ses inquiétudes liées à sa dépression. A la demande du superviseur, la résidente élabore un plan adéquat pour compléter le travail amorcé.
Les superviseurs démontrent ainsi un souci constant de coopération et de démarche d'amélioration de la compétence professionnelle des supervisés et une tolérance face aux hésitations et aux lenteurs de certains supervisés. Ils ajustent leurs stratégies selon les compétences, les difficultés, l'implication personnelle et l'accord des supervisés. Ils démontrent un souci d'efficacité et cherchent à optimiser la pertinence de leurs interventions. Ils évitent les escalades stériles dans les discussions, corrigeant rapidement tout écart de compréhension.

\begin{tabular}{|c|c|c|c|c|c|}
\hline \multicolumn{6}{|c|}{$\begin{array}{l}\text { Tableau } 2 \text { : } \\
12 \text { stratégies et } 22 \text { procédés utilisés par les superviseurs durant la supervision } \\
\text { en fonction du niveau et du champ d'intervention }\end{array}$} \\
\hline & & & \multicolumn{3}{|c|}{ Champs d'intervention } \\
\hline & & & $\begin{array}{l}\text { A. Formulation } \\
\text { du problème }\end{array}$ & B. Choix d'objectifs & $\begin{array}{l}\text { C. Moyens } \\
\text { d'investigation ou } \\
\text { d'intervention }\end{array}$ \\
\hline \multirow{5}{*}{ 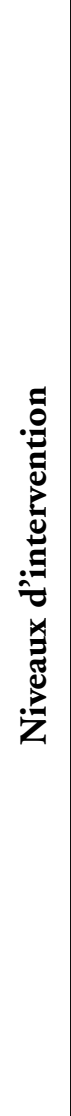 } & \multirow[b]{2}{*}{1} & \multirow[b]{2}{*}{$\begin{array}{l}\text { Gestion du processus } \\
\text { de supervision }\end{array}$} & $\begin{array}{l}\text { Structurer la } \\
\text { démarche** }^{* *}\end{array}$ & $\begin{array}{c}\text { Encadrer } \\
\text { le dialogue }\end{array}$ & $\begin{array}{c}\text { Instrumenter } \\
\text { dans la relation* }\end{array}$ \\
\hline & & & $\begin{array}{l}\text { - Introduire la supervi- } \\
\text { sion, s'entendre sur } \\
\text { une cible, annoncer la } \\
\text { méthode de travail..* }\end{array}$ & $\begin{array}{l}\text { - Identifier où on en } \\
\text { est par rapport à la } \\
\text { cible, annoncer ce }^{\text {qui suit. }}{ }^{* *}\end{array}$ & $\begin{array}{l}\text { - Exemple : proposer } \\
\text { un mini jeu de rôle } \\
\text { (superviseur dans la } \\
\text { position du } \\
\text { patient).* }\end{array}$ \\
\hline & \multirow{3}{*}{2} & \multirow{3}{*}{$\begin{array}{l}\text { Résolution du } \\
\text { problème à travers } \\
\text { la compétence du } \\
\text { résident }\end{array}$} & $\begin{array}{c}\text { Recueillir } \\
\text { les données*** }\end{array}$ & $\begin{array}{c}\text { Valider } \\
\text { leur pertinence* }\end{array}$ & Adapter à ce patient* \\
\hline & & & $\begin{array}{l}\text { - Interroger sur les faits, } \\
\text { accueillir les réponses } \\
\text { (réception)***, tester } \\
\text { les connaissances }(\mathrm{N}) \text {. }\end{array}$ & $\begin{array}{l}\text { - Faire anticiper à } \\
\text { l'aide des «si » *. }\end{array}$ & $\begin{array}{l}\text { - Faire planifier } \\
\text { l'intervention : } \\
\text { "Comment comptes- } \\
\text { tu procéder ?». * }\end{array}$ \\
\hline & & & $\begin{array}{l}\text { - Vérifier sa compréhen- } \\
\text { sion du problème en } \\
\text { reformulant ou en le } \\
\text { faisant résumer }(\mathrm{N}) .^{* *}\end{array}$ & $\begin{array}{l}\text { - L'inviter à se mettre } \\
\text { dans la peau du } \\
\text { patient }(\mathrm{N}) .^{*}\end{array}$ & \\
\hline
\end{tabular}




\section{Recherche et Perspectives}

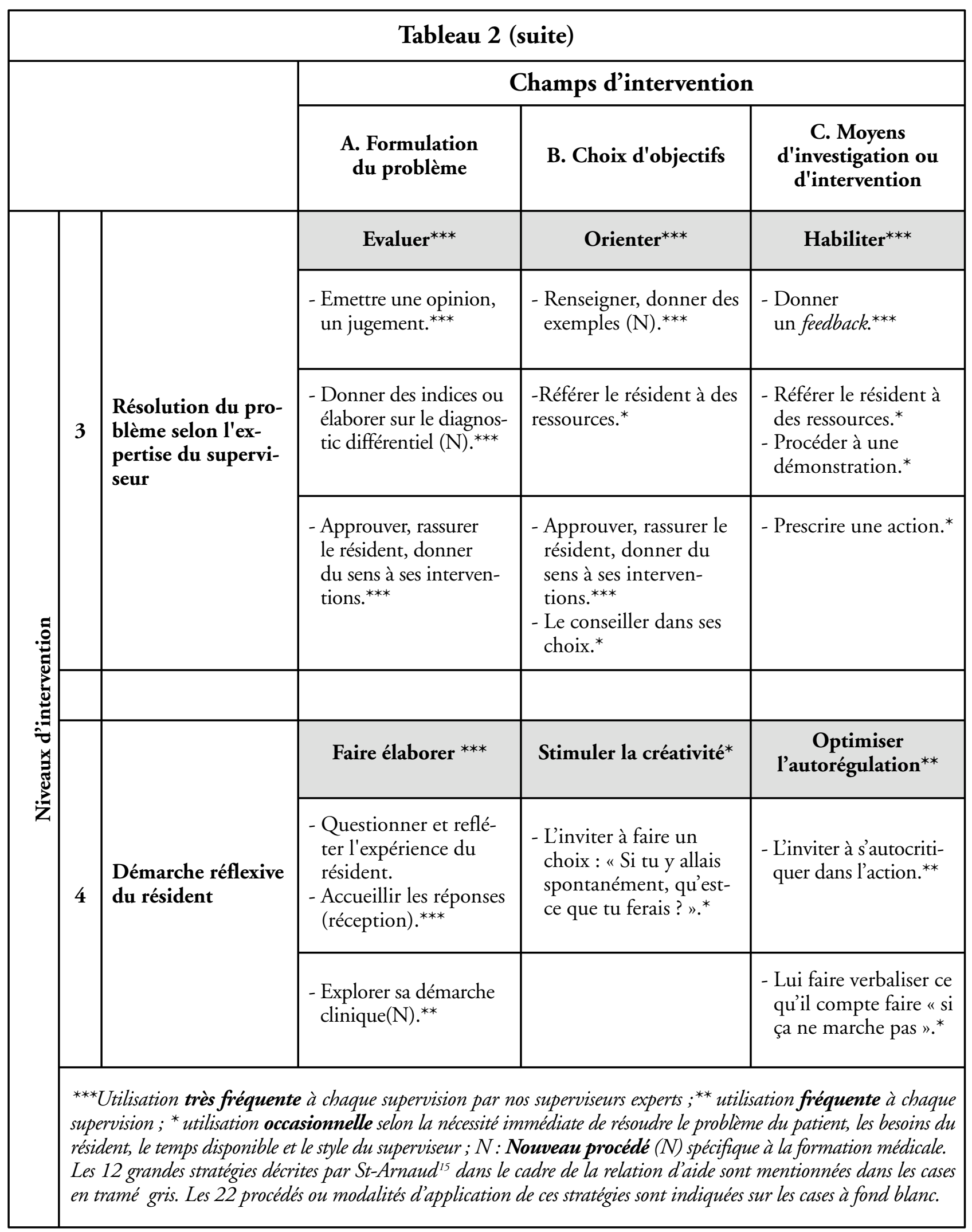




\section{Déroulement d'une demi-journée de supervision directe}

L'observation systématique de nos superviseurs experts en action permet de proposer un déroulement-type d'une demi-journée de supervision directe, comprenant les dix étapes décrites dans le tableau 3.

Ce déroulement-type peut servir de fiche d'observation ou d'auto-évaluation du superviseur.

\begin{tabular}{|c|c|c|c|}
\hline \multicolumn{4}{|c|}{$\begin{array}{l}\text { Tableau 3 : } \\
\text { Fiche d'auto-évaluation du superviseur en supervision directe }\end{array}$} \\
\hline Dix étapes lors d'une demi-journée de supervision* & Fait & $\begin{array}{l}\text { Fait en partie/mérite } \\
\text { amélioration (spécifier) }\end{array}$ & $\begin{array}{c}\text { Non } \\
\text { fait }\end{array}$ \\
\hline $\begin{array}{l}\text { Avant la supervision: } \\
\text { I. J'installe un cadre propice à la supervision en précisant son } \\
\text { contexte et en soignant le climat de la discussion (1). }\end{array}$ & & & \\
\hline $\begin{array}{l}\text { II. Je m'entends avec le résident sur une cible précise sur laquelle } \\
\text { portera le feedback à partir des questions qu'il se pose sur sa } \\
\text { performance, d'aspects qu'il souhaite améliorer (2), ou de ses } \\
\text { difficultés en lien avec sa performance antérieure (3). } \\
\text { J'annonce comment je compte procéder et vérifie ses sugges- } \\
\text { tions et son accord (1). }\end{array}$ & & & \\
\hline $\begin{array}{l}\text { A la suite immédiate d'une consultation: } \\
\text { III. Je lui demande de commenter sa performance (s'auto-éva- } \\
\text { luer), de préciser ce qui s'est passé (les faits) et ce qu'il en } \\
\text { pense (ses hypothèses) en lien avec la cible de travail (2). Au } \\
\text { besoin, je lui demande de décrire ce qu'il a bien fait et ce qu'il } \\
\text { ferait différemment la prochaine fois (4). }\end{array}$ & & & \\
\hline $\begin{array}{l}\text { IV. J'énonce ensuite mes observations (3) en spécifiant celles } \\
\text { qui concordent avec les siennes et celles qui s'ajoutent ou } \\
\text { sont différentes et je vérifie si mes observations lui semblent } \\
\text { " conformes " à ce qui s'est passé. }\end{array}$ & & & \\
\hline $\begin{array}{l}\text { V. Je pars de ces observations pour formuler mon opinion sur sa } \\
\text { performance (3) : explication de la conformité ou de l'écart } \\
\text { avec la performance attendue, en mettant l'accent sur ce qui } \\
\text { est « modifiable». Je lui propose des moyens concrets immé- } \\
\text { diats pour combler les lacunes identifiées et conclure adé- } \\
\text { quatement son entrevue avec le patient (3). }\end{array}$ & & & \\
\hline
\end{tabular}




\section{Recherche et Perspectives}

\section{Tableau 3 (suite)}

\begin{tabular}{|c|c|c|c|}
\hline Dix étapes lors d'une demi-journée de supervision* & Fait & $\begin{array}{l}\text { Fait en partie/mérite } \\
\text { amélioration (spécifier) }\end{array}$ & $\begin{array}{c}\text { Non } \\
\text { fait }\end{array}$ \\
\hline $\begin{array}{l}\text { En fin de séquence de supervision ou de demi-journée: } \\
\text { VI. Je lui propose de faire un retour sur les points identifiés }(2,3) \text { et } \\
\text { discute avec lui ce qui peut expliquer sa performance }(4) \text {. }\end{array}$ & & & \\
\hline $\begin{array}{l}\text { VII. Je fais un retour sur les moyens proposés au cours de la super- } \\
\text { vision et explore avec lui les moyens concrets pour corriger } \\
\text { l'écart de performance qui persiste }(2,3) \text { tout en discutant ce } \\
\text { qui pourrait rendre ce « changement difficile " (4). En com- } \\
\text { prend-il l'importance ou la pertinence? (4) Lui manque-t-il } \\
\text { des connaissances ou des outils pour le faire? (2) }\end{array}$ & & & \\
\hline $\begin{array}{l}\text { VIII. Je lui demande de résumer les changements (2) qu'il } \\
\text { compte réaliser (quoi et comment ?). Je l'amène à se don- } \\
\text { ner un plan d'action réaliste en précisant le «prochain pas } \\
\text { à franchir » et un échéancier raisonnable }(2,3) \text {. }\end{array}$ & & & \\
\hline $\begin{array}{l}\text { IX. Je reformule ce qu'il a fait spécifiquement pour réussir à } \\
\text { l'égard des points où il a atteint la performance attendue et } \\
\text { l'encourage à réutiliser ses réussites dans l'avenir (3). }\end{array}$ & & & \\
\hline $\begin{array}{l}\text { X. Je vérifie son niveau de satisfaction par rapport à notre dis- } \\
\text { cussion et lui demande des suggestions pour rendre le feedback } \\
\text { encore plus utile pour lui la prochaine fois (2). }\end{array}$ & & & \\
\hline${ }^{*}$ Les chiffres entre parenthèses se réferent aux niveaux d'intervention & & & \\
\hline
\end{tabular}




\section{Discussion}

Cette étude, utilisant une approche praxéologique, a le mérite d'ouvrir sur une méthode d'analyse des dialogues qui a démontré son efficacité dans d'autres champs professionnels ${ }^{18-19}$. Elle permet de comprendre les dialogues particuliers que les superviseurs entretiennent avec les étudiants qu'ils supervisent et de recenser les éléments issus de leur savoir pratique. Cette étude a permis de décrire, de catégoriser des niveaux, des champs d'intervention ainsi que des stratégies de supervision directe, à partir du cadre théorique décrit par St-Arnaud ${ }^{13-15}$ et de l'appliquer à la formation médicale. On a pu ainsi élargir l'éventail des stratégies utilisées en fonction des objectifs poursuivis par les superviseurs.

La clé du modèle proposé est l'intégration par le superviseur des quatre niveaux complémentaires d'intervention, qui détermine les stratégies et les procédés qui seront utilisés. Le niveau de la gestion du processus, rendu explicite par St-Arnaud ${ }^{14,15}$, suppose que le médecin superviseur soit proactif. Dès le début, il identifie avec le supervisé une cible commune de supervision, qu'il ajuste à partir de ses observations. Les superviseurs experts étudiés prennent un temps pour déterminer avec l'étudiant supervisé les objectifs de la supervision, comme le recommande le guide $\mathrm{n}^{\circ} 27$ de l'Association for Medical Education in Europe (AMEE) portant sur la supervision ${ }^{1}$. Ils assument l'encadrement nécessaire pour optimiser les apprentissages, non seulement au début mais tout au long de la supervision.

Les superviseurs privilégient dans leurs interventions le développement de la compétence du supervisé (niveau 2), tout en s'assurant grâce à leur expertise clinique de la qualité des soins dispensés au patient (niveau 3). En raison soit de la sévérité ou de la complexité du problème du patient, soit de l'importance des lacunes du supervisé, des contraintes de temps ou des limites de disponibilité du superviseur, ce dernier fait le choix de se centrer sur la gestion adéquate du cas médical et reporte à plus tard la discussion des apprentissages du supervisé. Ende et $a l^{20}$ mettent en évidence l'expérience de précepteurs en médecine interne qui, même en présence de lacunes importantes dans la gestion des problèmes médicaux, corrigent leurs internes en mettant l'accent sur les capacités d'auto-évaluation des apprenants et en utilisant des stratégies de questionnement qui, à l'aide d'indices, facilitent la découverte des réponses adéquates en vue d'augmenter leur confiance. Nos superviseurs experts privilégient aussi le développement de la compétence du résident en mettant l'accent sur les stra- tégies de facilitation du niveau 2. Lorsqu'ils utilisent le niveau 3 pour combler les lacunes du résident, ils limitent l'évocation de nouvelles informations à ce qui est nécessaire pour permettre à l'apprenant de gérer adéquatement la situation clinique et de poursuivre activement ses apprentissages. Procéder à une démarche réflexive (niveau 4) pour aller plus loin dans ses apprentissages est exigeant, car cela suppose une volonté de mieux comprendre ce qui se passe et une ouverture à apprendre à partir de ses erreurs ${ }^{21}$. Ceci souligne l'importance mise en évidence par nos superviseurs experts d'offrir cette opportunité à l'étudiant supervisé mais non de l'exiger.

Les résultats mettent en évidence l'importance des échanges entre le superviseur et le supervisé pour la formation professionnelle. Ils s'inscrivent en cohérence avec les derniers développements concernant les apprentissages en enseignement supérieur, tels qu'ils sont présentés par Huba et Freed ${ }^{11}$ ainsi que par Westberg et Jason ${ }^{7}$. Notre recherche y ajoute des stratégies plus fines que les superviseurs doivent maîtriser dans le décours de la supervision en médecine. Irby et ses collaborateurs ${ }^{22}$ ont identifié trois grandes stratégies pour améliorer l'enseignement, à savoir l'importance de la planification et de la préparation qui correspond au niveau 1 de la recherche, la réflexion sur les actions et les décisions prises qui correspond au niveau 4, détaillé par Pinsky et $a l^{23}$, et finalement le feedback. L'importance et les modalités du feedback en supervision sont résumées dans l'article d'O'Brien et al. ${ }^{9}$. Pour optimiser la rétroaction, le superviseur doit partir de l'auto-évaluation de l'apprenant ${ }^{7}$ pour donner son opinion sur la compétence de l'étudiant et lui proposer des conseils, des nouvelles informations ou des pistes d'exploration en vue soit de résoudre le problème du patient, soit d'aider l'apprenant à progresser dans le développement de sa compétence. Notre recherche rend explicite le niveau 2 axé sur la résolution du problème à travers la compétence de l'apprenant. Ce niveau donne un rôle actif à l'étudiant et encourage son autonomie, comme le soulignent Irby et $a .^{22}$. Les nouveaux éléments, amenés par l'expertise du superviseur au niveau 3, s'appuient alors sur les acquis de l'apprenant.

Toutefois, l'étude présente certaines limites. La grille utilisée, qui provient du champ de la consultation professionnelle et de la psychothérapie, peut avoir limité l'émergence de stratégies différentes du savoir pratique d'un superviseur en médecine. Cependant, nous avons obtenu rapidement une saturation des données, de sorte 


\section{Recherche et Perspectives}

que l'analyse des dernières supervisions n'apportait plus de nouvelles stratégies. L'autre limite est la proximité des chercheurs qui travaillaient dans le même milieu que les superviseurs experts. La méthode basée sur une analyse par consensus, la validation par un expert externe et les multiples triangulations des données pour confirmer les objectifs poursuivis par les superviseurs experts, compensent cette limite. Ende et al..$^{20}$ ont privilégié une méthode inductive pour mettre en évidence des stratégies liées à l'objectif pédagogique de correction des erreurs des apprenants. L'utilisation d'une méthode déductive, basée sur une approche praxéologique, a permis de préciser l'utilisation de stratégies pour atteindre divers objectifs pédagogiques, tels que la gestion du processus de supervision et le développement de la réflexivité des apprenants. Il va sans dire qu'il y a un risque d'oublier certains objectifs pédagogiques, mais aucune méthode ne peut garantir d'appréhender l'ensemble du processus de supervision.

Par ailleurs, les données proviennent d'un milieu québécois, fortement impliqué en pédagogie, où le temps consacré à la supervision est important. Toutefois, nous pensons que les résultats de notre étude sont applicables à différents milieux de supervision qui, comme le nôtre, ont la double contrainte de résoudre avec sécurité les problèmes de santé des patients rencontrés par les résidents en milieu ambulatoire et de développer la compétence de ces futurs médecins. Les niveaux explicités par notre recherche peuvent être appliqués à d'autres types de supervision. Cependant, le temps alloué à chacun des niveaux variera selon la charge clinique, le niveau 4 étant le plus à risque d'être négligé.

La généralisabilité de cette étude est limitée comme dans toutes études qualitatives, puisqu'elle ne porte que sur quelques supervisions dans le contexte spécifique de la formation en médecine familiale. Elle permet de comprendre les éléments du savoir pratique des superviseurs experts observés. Cependant, des études complémentaires sur le même thème sont nécessaires auprès de superviseurs novices pour préciser les différences de leurs pratiques avec celles des experts. Il serait également pertinent de cerner les stratégies utiles dans les différents types de supervision, notamment pour celles où le temps est plus limité, comme la supervision différée par discussion de cas, ou pour celles mises en oeuvres dans d'autres domaines que la médecine familiale. Comme la supervision est recommandée pour optimiser l'apprentissage des étudiants en médecine ${ }^{1}$, il y aurait lieu de vérifier si notre modèle s'applique aux interactions entre un enseignant et un apprenant de niveau prédoctoral, en se basant sur des outils différents de recueil des données, comme par exemple le portfolio.

\section{Conclusion}

Cette recherche visait à préciser le savoir pratique des superviseurs experts selon l'approche praxéologique développée par Schon et Argyris ${ }^{18,19}$. En partant du modèle de St-Arnaud ${ }^{13-15}$, cette recherche qualitative a permis de rendre compte des différents objectifs poursuivis par les superviseurs experts en formation de médecine familiale. Quoique non standardisée, l'approche développée à la suite de notre recherche propose un modèle explicite de collaboration en supervision. Ce modèle permet de tenir compte de la sécurité du patient et de l'apprenant, tout en aidant au développement de sa compétence. Il est utile pour l'identification des besoins de formation professorale. Il outille les enseignants avec un ensemble de stratégies qui tiennent compte des divers objectifs pédagogiques qu'ils poursuivent. Cela rend le superviseur plus efficient. A l'aide de ce modèle, des ateliers de formation professorale sur le processus de supervision ont été développés à différents niveaux de la formation médicale pour favoriser un travail de collaboration avec les apprenants.

\section{Contributions}

Bernard Martineau et Gilles Girard ont contribué collégialement et solidairement à la conception de l'étude, au recueil et à l'analyse des données et à la rédaction du manuscrit. Richard Boulé, qui est un des trois superviseurs cliniciens experts de l'étude, s'est joint aux chercheurs principaux au moment de la validation des analyses et a participé activement à la rédaction du manuscrit. Les auteurs remercient Yves St-Arnaud qui a agi comme consultant expert externe pour valider les cotations et les catégorisations des chercheurs. Il a aussi lu le manuscrit et a jugé qu'il traduisait adéquatement l'approche praxéologique. 


\section{Références}

1. Kilminster S, Cottrell D, Grant J, Jolly B. AMEE Guide No 27: Effective educational and clinical supervision. Med Teach 2007;29:2-19.

2. Kilminster S, Jolly B. Effective supervision in clinicl practice settings: a litterature review. Med Educ 2000; 34:827-80.

3. Bernard JM, Goodyear RK. Fundamentals of Clinical Supervision. Boston: Allyn and Bacon, 1992.

4. Martineau B. L'enseignement de la relation médecinmalade en médecine familiale à l'aide de l'observation directe: étude de cas d'une interaction superviseursupervisé. Mémoire de maîtrise. Université Laval (Québec), 1993.

5. Saucier D. Direct observation of residents in family medicine: a qualitative study of four practices. Mémoire de maitrise, Faculté des études graduées, Université Laval (Québec), avril 1994.

6. Beaumier A, Bordage G, Saucier D, Turgeon J. Nature of the clinical difficulties of first-year family medicine residents under direct observation. CMAJ 1992;146:489-97.

7. Westberg J. Jason H. Fostering reflection and providing feedback. Helping others learn from experience, NewYork: Springer series in medical education, 2001.

8. Ende J. Feedback in Medical Education. JAMA 1983; 250:771-81.

9. O'Brien HV, Marks MB, Charlin B. Le feedback: un élément essentiel de l'intervention pédagogique en milieu clinique. Pédagogie Médicale 2003;4:184-91.

10. Irby DM. What clinical teachers in medicine need to know. Acad Med 1993;69:333-42.

11. Huba M. Freed J. Learner-centered assessment on college campus. Shifting the focus from teaching to learning, Boston: Allyn and Bacon, 2000.

12. Christensen CR. Every students teaches and every teacher learns: The reciprocal gift of discussion teaching. In: Christensen CR, Garvin A et Sweet A, Education for judgment: The artistry of discussion leadership, Boston: Harvard Business School Press, 1991,99119.
13. St-Arnaud Y. Connaître par l'action. Montréal : Les Presses de l'Université de Montréal, 1992.

14. St-Arnaud Y. L'interaction professionnelle: Efficacité et coopération. Montréal: Les Presses de l'Université de Montréal, 1995.

15. St-Arnaud Y. Relation d'aide et psychothérapie. Le changement personnel assisté. Boucherville: Gaëtan Morin Éditeur, 2001.

16. Fraenkel JR, Wallen NE. How to design and evaluate research in education. Boston: McGraw-Hill, 2003.

17. Chamberland $M$, Hivon R. Les compétences de l'enseignant clinicien et le modèle de rôle en formation clinique. Pédagogie Médicale 2005;6:98-111.

18. Argyris C, Schön DA. Theory in practice: increasing professional effectiveness. San Francisco: Jossey-Bass, 1974.

19. Schön DA. The reflective practitionner: How professionnals think in action. New York: Basic books, 1983.

20. Ende J, Pomerantz A, Erikson F. Preceptors' strategies for correcting residents in an ambulatory care medicine setting: a qualitative analysis. Acad Med 1995; 70:224-9.

21. Neufeldt SA, Karno MP, Nelson ML. A qualitative study of experts, conceptualization of supervisee reflectivity. Journal of Counseling Psychology 1996;43:3-9.

22. Irby DM, Ramsey PG, Gillmore GM, Scaad D. Characteristics of effective clinical teachers of ambulatory care medicine. Acad Med 1991;66:54-5.

23. Pinsky LE, Monson D, Irby DM. How excellent teachers are made: Reflecting on success to improve teaching. Adv. Health Sci Educ Theory Pract 1998;3:207-15.

24. Usatine RF, Nguyen K, Randall J, Irby DM. Four exemplary preceptors' strategies for efficient teaching in managed care settings. Acad Med, 1997;72:766-9.

Manuscrit reçu le 5 juin 2007 ; commentaires éditoriaux formulés aux auteurs le 31 décembre 2007 ; accepté pour publication le 15 janvier 2008 